\section{Facharztprüfung zur Erlangung des Facharzttitels FMH für Allergologie und Klinische Immunologie}

Aufgrund des Prüfungsreglementes, welches am 1. Juli 1995 in Kraft gesetzt wurde, ist die Teilnahme an der Facharztprüfung für diejenigen Kandidatinnen und Kandidaten Voraussetzung für die Erlangung des Facharzttitels Allergologie und Klinische Immunologie, welche ihre Weiterbildung in Allergologie und Klinischer Immunologie bis am 30. Juni 1996 nicht abgeschlossen hatten. Es empfiehlt sich, die Facharztprüfung frühestens im letzten Jahr der reglementarischen Weiterbildung abzulegen (Art. 23 WBO).
Ort: Inselspital, Allergologisch-immunologische Poliklinik, Bern.

Datum: Donnerstag, 7. September 2000.

Prüfungsgebühr: Die Fachgesellschaft erhebt eine Prüfungsgebühr von Fr. 500.-.

\section{Anmeldung:}

Sekretariat SGAI, Frau Therese Kaspar,

Gryphenhübeliweg 40, Postfach, 3000 Bern 6, Tel. 03135990 91, Fax 03135990 92,

E-Mailsgai@bluewin.ch

Anmeldefrist: 31. Mai 2000

\section{Examen de spécialiste en vue de l'obtention du titre de spécialiste FMH en allergologie et immunologie clinique}

Conformément au règlement d'examen qui est entré en vigueur le $1^{\text {er }}$ juillet 1995 , la participation à l'examen de spécialiste est une condition requise pour les candidats à l'obtention du titre de spécialiste FMH allergologie et immunologie clinique qui n'avaient pas terminé leur formation postgraduée en allergologie et immunologie clinique le 30 juin 1996. Il leur est recommandé de passer l'examen de spécialiste au plus tôt durant la dernière année de leur formation postgraduée réglementaire (art. 23 RFP).
Lieu: Hôpital de l'Ile, Policlinique d'allergologie et d'immunologie, Berne.

Date: Jeudi, le 7 septembre 2000.

Taxe d'examen: La société de discipline médicale prélève une participation aux frais de Fr. 500.-.

\section{Inscription:}

Secrétariat SSAI, Madame Therese Kaspar,

Gryphenhübeliweg 40, case postale, 3000 Berne 6, tél. 03135990 91, fax 03135990 92,

e-mail sgai@bluewin.ch

Délai d'inscription: le 31 mai 2000 